\title{
A Comparative Study for Modelling the Survival of Breast Cancer Patients in the West of Iran
}

\author{
Mozhgan Safe ${ }^{1}$, Hossein Mahjub ${ }^{1,2} \&$ Javad Faradmal ${ }^{1,3}$ \\ ${ }^{1}$ School of Public Health, Hamadan University of Medical Sciences, Hamadan, Iran \\ ${ }^{2}$ Research Center for Health Sciences, Hamadan University of Medical Sciences, Hamadan, Iran \\ ${ }^{3}$ Modeling of Noncommunicable Disease Research Center, Hamadan University of Medical Sciences, Hamadan, \\ Iran \\ Correspondence: Javad Faradmal, School of Public Health, Hamadan University of Medical Sciences, Hamadan, \\ Iran. E-mail: javad.faradmal@umsha.ac.ir
}

Received: April 7, 2016 Accepted: June 25, 2016 Online Published: July 6, 2016

doi:10.5539/gjhs.v9n2p215 URL: http://dx.doi.org/10.5539/gjhs.v9n2p215

\begin{abstract}
Background: Breast cancer is the main cause of women cancer mortality. Therefore, precise prediction of patients' risk level is the major concern in therapeutic strategies. Although statistical learning algorithms are high quality risk prediction methods, but usually their better prediction quality leads to more loss of interpretability. Therefore, the aim of this study is to compare 'Model-Based Recursive Partitioning' and 'Random Survival Forest'; whether the partitioning, as the more interpretable learning technique, could be a suitable successor for forests.
\end{abstract}

Patients and Methods: The applied dataset for this retrospective cohort study includes the information of 539 Iranian females with breast cancer. To model the patients' survival, various learning algorithms were fitted and their accuracy measures were statistically compared by means of several precision criteria.

Results: This study verified the stability of 'Model-based Recursive Partitioning', further to 'Random Survival Forest' deficiency to present a unique pervasive model. Moreover, except 'Log-Logistic-Based Recursive Partitioning', none of the methods significantly outperformed 'Exponential- Based Recursive Partitioning'.

Conclusions: Briefly, it was concluded that the loss of interpretability due to the use of over complex models, may not always counterbalanced by the amount of prediction improvements.

Keywords: breast cancer, model-based recursive partitioning, random survival forest

\section{Background}

Breast cancer as the most common cancer among Iranian females (Aboutorabi, Hadian, Ghaderi, Salehi, \& Ghiasipour, 2015), is word widely the $18 \%$ proportional share of all cancers in women (Cvetković \& Nenadović, 2016). Certainly, the prerequisites for the disease prevention and proficient treatment findings are the precise detection of affective risk factors in diseases formation and progression (Mert, Kilıç, Bilgili, \& Akan, 2015). For a long time ago, conventional survival models have been used to achieve this aim and to provide necessary requisites for breast cancer prognostication (Ahmadinejad, Movahedinia, Movahedinia, Naieni, \& Nedjat, 2013; Azarkish, Najmabadi, Roudsari, \& Shandiz, 2015; Sadoughi, Afshar, Olfatbakhsh, \& Mehrdad, 2016). Although the prominent interpretability of these models has made them pioneer for simple medical explanations, but the lower bias and accurate prediction of recently introduced learning algorithms, has stimulated the statistical focus on machine learning methods. The superior performance of these state-of-the-art learning techniques has been confirmed previously in many medical studies (Chao, Yu, Cheng, \& Kuo, 2014; Dezfuly \& Sajedi, 2015; Habibi, Ahmadi, \& Alizadeh, 2015).

'Model-Based Recursive Partitioning' (MoBRP) could be referred as an exemplary interpretable machine learning technique (Zeileis, Hothorn, \& Hornik, 2008a). Actually, this partitioning is a hybrid tree such that combines the traditional parametric survival models with newly introduced recursive partitioning methods (Zeileis et al., 2008a). By this way, MoBRP gains the profits of both classical and modern analysis; the prominent interpretability for recognized affective risk factors, in addition to its accurate survival time prediction 
and modeling.

Further to MoBRP, 'Random Survival Forest' (RSF) could be referred as another outstanding learning technique (Ishwaran, Kogalur, Blackstone, \& Lauer, 2008). In fact, this more intricate and time-consuming repetitious algorithm is an ensemble of survival trees and therefore would be so expert to find high order effects and interactions (Hastie, Tibshirani, Friedman, \& Franklin, 2005).

Although the MoBRP ability to recognize nonlinearity and high order interactions, has made it superior to ordinary parametric survival models, but its detection ability for more complicated structures is hierarchically inferior to RSF. Briefly, more precise prognostication requires more costly computational analysis which usually provides less interpretable results; whereas, many publications have certified that the loss of interpretability due to the use of over complex algorithms may not always counterbalanced by the amount of prediction improvements (Haibe-Kains, Desmedt, Sotiriou, \& Bontempi, 2008).

The RSF performance has been evaluated several times previously. For instance comparative studies, using both of RSF and Cox proportional hazard, for modeling the survival of patients with different cancers as breast (Kurt Omurlu, Ture, \& Tokatli, 2009), prostate (Gerds, Kattan, Schumacher, \& Yu, 2013), head and neck (Datema et al., 2012), as well as patients with systolic heart failure (Hsich, Gorodeski, Blackstone, Ishwaran, \& Lauer, 2011). Forests were also compared with variety of learning techniques (Mirmohammadi, Shishehgar, \& Ghapanchi, 2014; Pang, Datta, \& Zhao, 2010) and survival trees, as the forest elements (Yosefian, Mosa Farkhani, \& Baneshi, 2015); but as far as we know, the RSF has never been compared with MoBRP. Therefore, the aim of this study is to compare the accuracy of predictions obtained with MoBRP and RSF; as if MoBRP which is more interpretable technique, could be a suitable successor for computationally expensive RSF.

\section{Patients and Methods}

\subsection{Patients}

In this retrospective cohort study, the information of 539 eligible women with breast cancer was gathered. All the involved patients have undergone, at least, one surgery for tumor extraction, from 1995 to 2013. The surgeries were under the supervision of the Diagnostic Center of Darolaytam-e Mahdieh of Hamedan, as the referential center in the west of Iran.

The interested event was death of breast cancer and the survival time was measured in days from surgery to death. Almost, $63 \%$ of patients were censored and had never experienced the event of interested, in the follow up duration.

\subsection{Model-Based Recursive Partitioning Algorithm}

Simply, MoBRP is a classification tree that is capable for parametric model fitting in each node of the tree (Zeileis et al., 2008a). Therefore, participated variables in MoBRP could be considered for two distinct objectives: (i) partitioning variables which are used for grow trees and forming the terminal nodes and (ii) model variables, which are used for explain the survival time in each node. It is worth noting that; these two types of variables could partially or totally be the same.

In order to grow this special tree, each node would be partitioned if the instability of the fitted model associated to it, is statistically significant through some partitioning variables; more precisely, for all terminal nodes and through each partitioning variable, the stabilities of models are assessed and the variable which is responsible for the most instability is selected as partitioning variable for its associated terminal node. Additionally, the cut point selection would be in favor of some objective functions to globally optimize the models of terminal nodes (Zeileis et al., 2008a)

\subsection{Random Survival Forests Algorithm}

The conclusion of a RSF algorithm is simply the average of its constituent trees. To grow a forest including B trees, the following steps are repeated B times to produce each tree (Hastie et al., 2005; Ishwaran et al., 2008).

A random sample with replacement, and of size $\mathrm{N}$, is drowning from the original observations. At each node of the tree, $\mathrm{p}$ covariates are randomly selected and the partitioning would be based on the covariate which can provide the largest survival differences between generated nodes. Note that the covariate and its cut point are selected by a heuristic search through the all terminal nodes and for each possible split point along. Furthermore, different criterion could be used for this difference assessment (Hastie et al., 2005; Ishwaran et al., 2008).

The growing would continue until a tree reaches its constrain about the least permissible number of observations at terminal nodes. 


\subsection{Performance Assessment}

In order to compare the efficiency of the aforementioned risk prediction methods, the five most common accuracy measures, for nonparametric survival algorithms, were used: the time-dependent receiver operating characteristic (ROC) curve (Heagerty, Lumley, \& Pepe, 2000), the sensitivity and specificity, the concordance index (Harrell, Lee, \& Mark, 1996), the Brier score (Brier, 1950; Graf, Schmoor, Sauerbrei, \& Schumacher, 1999) and the hazard ratio (HR) from Cox's proportional hazards model (Cox, 1972).

\subsection{Analysis Framework}

In this study, the MoBRP was grown based on the four most common parametric survival models (Corbiere \& Joly, 2007); as: Exponential, Weibull, Log-Logistic and Log-Normal.

The applied growing rules for RSF were Log-Rank and Log-Rank Score, that is, node splitting through maximization of log-rank and its standardized statistics, respectively (Ishwaran et al., 2008).

To derive MoBRP and RSF risk group prediction, the patients with the lower $33 \%$ of the predicted risk score were considered as low risk; and the remains as high risk groups. It should be mentioned; this experimental proportion has been certified in various breast cancer prognostications (Buyse et al., 2006; Haibe-Kains et al., 2008).

Since exponential is the simplest parametric model, it was selected as the benchmark for MoBRP, whether the more complexity of other methods can provide them superior prognostications. All the comparisons were statistically tested except the values of sensitivity and specificity; due to the lack of any proven statistical test.

Lastly, although the train and test sets were randomly selected from the dataset, but in order to demystify the style of subsets selection, all the analyses were repeated substituting the sets (Michiels, Koscielny, \& Hill, 2005).

\section{Results}

The patients' median lifetime was 8.85 years and the 5-year survival rate was $68 \%$ (95\%CI: $64 \%-72 \%)$. The longest observed follow up duration was approximately 19 years. The variable's importance, provided by both of forests, demonstrated the 'Progesterone Receptor Status' (PR) and 'Number of Involved Lymph Nodes', respectively as the two most relevant variables to explain the survival duration. These variables were also significant in all MoBRPs.

Table 1 represents the sensitivities and specificities for risk group predictions. Excepting Exponential-BRP, almost all sensitivities were more than 0.7. Although the most sensitivity were associated to RSFs, but they were able to provide more sensitivities only for train set and MoBRP methods were pioneer in the test set. Furthermore, the specificities of the forests were lowest for test set, in spite of their moderate specificities for train set. Also, Exponential-BRP showed the most specificity despite its lowest sensitivities.

Table 1. Sensitivity and specificity for risk group prediction for each of the train and test set

\begin{tabular}{lllll}
\hline \multirow{2}{*}{ Model } & \multicolumn{3}{l}{ Sensitivity } & \multicolumn{3}{l}{ Specificity } \\
\cline { 2 - 5 } & train & test & train & test \\
\hline Exponential-BRP & 0.597 & 0.510 & 0.624 & 0.702 \\
Weibull-BRP & 0.747 & 0.779 & 0.461 & 0.496 \\
Log-Logistic-BRP & 0.819 & 0.789 & 0.417 & 0.494 \\
Log-Normal-BRP & 0.787 & 0.791 & 0.431 & 0.438 \\
RSF Log-Rank & 0.933 & 0.678 & 0.465 & 0.339 \\
RSF Log-Rank Score & 0.945 & 0.716 & 0.458 & 0.354 \\
\hline
\end{tabular}

M-BRP: Model-Based Recursive Partitioning.

Addition to C-index and IBS, which are common for risk score and group assessments, IAUC and HR have been included in Tables 2 and 3, respectively. The results of risk score prediction were confirmed by the results of risk group prediction, through the signification of statistical tests involving C-indices and IBSs. Excluding IAUCs, almost none of the MoBRPs were significantly more accurate than Exponential-BRP, for each of the measures or each of the train or test sets. Moreover, RSFs were in agreement and their superiorities were declared, unanimously by all the measures; however, their excellence was specific to train set and they failed to significantly outperform in the test set. This paradox could also be seen for all the prediction methods, according to IAUCs. 
Table 2. Performance for risk score prediction for each of the train and test set

\begin{tabular}{lllllll}
\hline \multirow{2}{*}{ Model } & \multicolumn{1}{l}{$C$-index } & & IAUC & \multicolumn{3}{l}{ IBSC } \\
\cline { 2 - 7 } & train & test & train & test & train & test \\
\hline Exponential-BRP & 0.653 & 0.625 & 0.667 & 0.623 & 0.183 & 0.219 \\
Weibull-BRP & 0.672 & 0.615 & $0.658^{* *}$ & 0.615 & 0.194 & $0.215^{* *}$ \\
Log-Logistic-BRP & 0.681 & 0.609 & $0.669^{* *}$ & 0.617 & $0.168^{* *}$ & $0.219^{* *}$ \\
Log-Normal-BRP & 0.686 & 0.615 & $0.686^{* *}$ & 0.613 & 0.195 & 0.221 \\
RSF Log-Rank & $0.761^{* *}$ & 0.556 & $0.773^{* *}$ & 0.569 & $0.177^{* *}$ & 0.237 \\
RSF Log-Rank Score & $0.755^{* *}$ & 0.556 & $0.763^{* *}$ & 0.559 & $0.183^{* *}$ & 0.229 \\
\hline
\end{tabular}

M-BRP: Model-Based Recursive Partitioning; *Significant at 5\%; **Significant at 1\%; All the tests were designed as if the accuracy of every method is more than Exponential-BRP.

Table 3. Performance for risk group prediction for each of the train and test set

\begin{tabular}{lllllll}
\hline \multirow{2}{*}{ Model } & \multicolumn{2}{l}{-index } & & HR & \multicolumn{3}{l}{ IBSC } \\
\cline { 2 - 7 } & train & test & train & test & train & test \\
\hline Exponential-BRP & 0.680 & 0.657 & 1.96 & 1.57 & 0.182 & 0.171 \\
Weibull-BRP & 0.707 & 0.706 & 2.05 & 2.22 & 0.148 & $0.161^{* *}$ \\
Log-Logistic-BRP & 0.762 & 0.705 & 2.47 & 2.18 & 0.147 & $0.162^{* *}$ \\
Log-Normal-BRP & 0.752 & 0.682 & 2.37 & 1.93 & 0.147 & 0.170 \\
RSF Log-Rank & $0.869^{* *}$ & 0.527 & $3.87^{*}$ & 1.00 & $0.182^{* *}$ & 0.177 \\
RSF Log-Rank Score & $0.850^{* *}$ & 0.587 & $3.19^{*}$ & 1.20 & $0.184^{* *}$ & 0.174 \\
\hline
\end{tabular}

M-BRP: Model-Based Recursive Partitioning; *Significant at 5\%; **Significant at $1 \%$; All the tests were designed as if the accuracy of every method is more than Exponential-BRP.

Log-Logistic-BRP was the sole method which its risk score predictions outperformed, in both of test and train sets; though, this supremacy seems uncertain as only one measure, i.e. IBS, affirmed that. However, Log-Logistic-BRP has performed leastwise similar to other methods; therefore, another generic Log-Logistic-BRP was fitted by participation of all the observations in order to provide a pervasive infrastructure for physicians. In this implementation, a four-terminal-node tree was formed. The percent of high-risk patients according to risk group prediction were respectively, $17 \%, 34 \%, 67 \%$ and $85 \%$, associated to each terminal node; and the median lifetimes were 108, 100, 98 and 89 months, correspondingly.

The stability of conclusions was confirmed by achieving the similar results after the replacement of training and test sets. As before, Exponential-BRP had the least sensitivity; however, it demonstrated most specificity in risk prediction. Although all the assessment criteria statistically certified more accuracy of RSF for this new training set, but none of them could discover any more precise for new test set.

\section{Discussion}

Since for none of the assessment measures, RSF significantly outperformed the Exponential-BRP in both of train and test sets; this investigation certified that the probable accuracy improvement caused by costly computational RSF does not compensate the loss of interpretability. The superiority of RSF only for its training set, testifies the RSF over-fitting and its deficiency to provide a generic pervasive model. Consistent with this conclusion, the RSF over-fitting has been cited as its disadvantage by many previous medical studies (Mirmohammadi et al., 2014). Whereas, other studies (Ishwaran, 2007) which have compared RF with Classification and Regression Trees, have claimed the RF more capability for over-fit controlling. The idea of seldom over fitting with random forest classification is also affirmed by other documentations (Hastie et al., 2005).

Considering Log-Logistic-BRP, the present order for the percentage of high-risk patients and the median lifetime related to terminal nodes clearly attests the ability of tree to divide the population to homogeneous subsets with the same risk levels. Therefore, in addition to MoBRP regression-structure, its excellent classification-structure should also be highlighted. Meanwhile, it is cited that the results of RSF would not be easy to interpret for clinicians, due to RSF inability for any classification (Walschaerts, Leconte, \& Besse, 2012).

There are many studies which have estimated the risk factor importance of breast cancer by means of RSF. In line with our findings Ishwaran et al. and Kurt Omurlu et al. reported the importance of 'PR' and 'Number of 
Nodes'; but in contrast with us, they found 'Number of Nodes' as a more important risk factor (Ishwaran et al., 2008; Kurt Omurlu et al., 2009). In spite of these diagnosed factors, it has been claimed that the predicted importance by RSF is bias due to its bias of variable selection (Strobl, Boulesteix, Zeileis, \& Hothorn, 2007). To explain more, note that other than MoBRP which is a special unbiased tree, there are numerous studies that have discussed the bias of variable selection in the algorithm of usual tree models (Hothorn, Hornik, \& Zeileis, 2006; Kim \& Loh, 2001). Clearly, a forest composed of bias trees would lead to bias predictions of variable importance.

Addition to the mentioned factors, all the MoBRPs recognized the significant effects for 'Tumor Size'; since the adverse effect of this factor was previously certified in many clinical researches (Faradmal, Soltanian, Roshanaei, Khodabakhshi, \& Kasaeian, 2014); therefore, MoBRPs seems to be more able for risk factor recognition.

In partial agreement with our study, D'Eredita cited 'Lymph Status', 'Tumor Size' and 'Histological Grade' as the most informative medical factors (D'Eredita, Giardina, Martellotta, Natale, \& Ferrarese, 2001). Also, the study of Delen et al. (Delen, Walker, \& Kadam, 2005) affirmed the effectiveness of 'Tumor Size' through the sensitivity analysis of Artificial Neural Network. In agreement to our results, they found 'Number of Involved Lymph Nodes' as another important factor but in contrast to ours, they reported the 'Stage of Disease' as a more informative index. In their study decision tree showed the best prediction following with Artificial Neural Network and Logistic Regression. They also suggested that modern pattern recognition tools should be used as the complementary for traditional statistical modelling. It is worth noting that, their idea could be considered as a reference for MoBRP which combines the algorithm of both methods.

The prediction quality of RSF has been appraised many times. Bou-Hamad et al. assessed the RSF performance for predicting the survival of patients with primary biliary cirrhosis of the liver. The resulted IBS from a 10-fold cross validation certified the best implementation for RSF following with bagging (Bou-Hamad, Larocque, \& Ben-Ameur, 2011). In another real data application to model the survival time of Iranian females with breast cancer, random forest showed the highest level of accuracy among other learning techniques (Montazeri, Montazeri, Montazeri, \& Beigzadeh, 2015). In spite of mentioned studies in the context of learning algorithms, there are also comparisons between RSF and Cox, as the most widely used method for modeling the censored data (Hothorn, Bühlmann, Dudoit, Molinaro, \& Van Der Laan, 2006). Through some of these comparative studies Cox has shown, not only the same (Hsich et al., 2011), but also better performance than all diversity of forests (Datema et al., 2012). Additionally, the supremacy of Cox has been confirmed by other simulation studies (Kurt Omurlu et al., 2009). Evidently and in agreement to our findings, random forest is so prominent among learning algorithms; however, its computational complexity may not always guarantee its superiority over traditional models.

Other than MoBRP, random forest has been also compared with survival trees with so simpler structure. In a study which was designed to model the survival of Iranian patients suffering from acute myocardial infarction, both of IBS and C-index ascertained the RSF more precise results; furthermore, the difference between indices in training and test sets, evidenced the more generalizability of the forest (Yosefian et al., 2015). It should be added that the RSF less prediction error rates, has been also proven in another study in the field of breast cancer survival modeling (Walschaerts et al., 2012). Maroco et al. compared the performance of seven data mining classifiers in addition to three traditional models including logistic regression. They concluded that the overall accuracy of random forest out performed three different types of classification trees and logistic regression (Maroco et al., 2011). Eventually, although forest outperforms each of its constituent trees but our study statistically certified that MoBRP, as a less complex algorithm, can provide sufficient accuracy and less over-optimization.

For none of the aforementioned studies, statistical test was used to compare the accuracy measures. In actuality, the investigation of Haibe-Kains et al. is referring as the first study that has statistically compared the performance of learning algorithms for breast cancer prognostication from gene expression data (Haibe-Kains et al., 2008). In accordance with the present study, they claimed that the loss of interpretability as the consequences of complex models does not equilibrate the provided excess prices in breast cancer prognostication (Haibe-Kains et al., 2008).

It should be noted that the retrospective design of this study could be referred as its main limitation. Although, it was preferred to conduct a study including all Iranian females with breast cancer, but the target population of this survey only includes breast cancer patients from the west of Iran. Finally, regarding to the high observed censoring rate and the high possibility of being cured, variety of cure models seem to be a proper proposal for survival modelling and more investigations. 


\section{Conclusion}

Briefly, more precise prognostication is in the wake of more costly computational analysis which usually provides less interpretable recognitions. This study certified MoBRP as a compromise between a high quality prediction and the ease of interpretation for clinicians; therefore, a good analytical model, in medical fields.

\section{Acknowledgments}

This investigation which is a part of Ph.D. thesis; is resulted by the fund of Vice Chancellor for Research and Technology of Hamadan University of Medical Sciences. We appreciate them and also the Diagnostic Center of Hamedan Mahdieh Darolaytam for helping us in collecting data.

\section{Competing Interests Statement}

The authors declare that there is no conflict of interests regarding the publication of this paper.

\section{References}

Aboutorabi, A., Hadian, M., Ghaderi, H., Salehi, M., \& Ghiasipour, M. (2015). Cost-effectiveness analysis of trastuzumab in the adjuvant treatment for early breast cancer. Global Journal of Health Science, 7(1), 98-106. http://dx.doi.org/10.5539/gjhs.v7n1p98

Ahmadinejad, N., Movahedinia, S., Movahedinia, S., Naieni, K. H., \& Nedjat, S. (2013). Distribution of breast density in Iranian women and its association with breast cancer risk factors. Iranian Red Crescent Medical Journal, 15(12), e16615. http://dx.doi.org/10.5812/ircmj.16615

Azarkish, F., Najmabadi, K. M., Roudsari, R. L., \& Shandiz, F. H. (2015). Factors Related to Return to Work in Women After Breast Cancer in Iran. Iranian Red Crescent Medical Journal, 17(9), e19987. http://dx.doi. org/10.5812/ircmj. 19978

Bou-Hamad, I., Larocque, D., \& Ben-Ameur, H. (2011). A review of survival trees. Statistics Surveys, 5, 44-71. http://dx.doi.org/10.1214/09-SS047

Brier, G. W. (1950). Verification of forecasts expressed in terms of probability. Monthly Weather Review, 78(1), 1-3. http://dx.doi.org/10.1175/1520-0493(1950)078<0001:VOFEIT>2.0.CO;2

Buyse, M., Loi, S., Van't Veer, L., Viale, G., Delorenzi, M., Glas, A. M., ... Ellis, P. (2006). Validation and clinical utility of a 70-gene prognostic signature for women with node-negative breast cancer. Journal of the National Cancer Institute, 98(17), 1183-1192. http://dx.doi.org/10.1093/jnci/djj329

Chao, C.-M., Yu, Y.-W., Cheng, B.-W., \& Kuo, Y.-L. (2014). Construction the Model on the Breast Cancer Survival Analysis Use Support Vector Machine, Logistic Regression and Decision Tree. Journal of Medical Systems, 38(10), 1-7. http://dx.doi.org/10.1007/s10916-014-0106-1

Corbiere, F., \& Joly, P. (2007). A SAS macro for parametric and semiparametric mixture cure models. Comput Methods Programs Biomed, 85(2), 173-180. http://dx.doi.org/10.1016/j.cmpb.2006.10.008

Cox, D. R. (1972). Regression models and life-tables. Journal of the Royal Statistical Society [Series B], 34, $187-220$.

Cvetković, J., \& Nenadović, M. (2016). Depression in breast cancer patients. Psychiatry Research, 240, 343-347. http://dx.doi.org/10.1016/j.psychres.2016.04.048

D’Eredita, G., Giardina, C., Martellotta, M., Natale, T., \& Ferrarese, F. (2001). Prognostic factors in breast cancer: The predictive value of the Nottingham Prognostic Index in patients with a long-term follow-up that were treated in a single institution. European Journal of Cancer, 37(5), 591-596. http://dx.doi.org/10. 1016/S0959-8049(00)00435-4

Datema, F. R., Moya, A., Krause, P., Bäck, T., Willmes, L., Langeveld, T., ... Blom, H. M. (2012). Novel head and neck cancer survival analysis approach: Random survival forests versus cox proportional hazards regression. Head \& Neck, 34(1), 50-58. http://dx.doi.org/10.1002/hed.21698

Delen, D., Walker, G., \& Kadam, A. (2005). Predicting breast cancer survivability: A comparison of three data mining methods. Artificial Intelligence in Medicine, 34(2), 113-127. http://dx.doi.org/10.1016/j.artmed.2004. 07.002

Dezfuly, M., \& Sajedi, H. (2015). Predict Survival Of Patients With Lung Cancer Using An Ensemble Feature Selection Algorithm And Classification Methods In Data Mining. Journal of Information, 1(1), 1-11. http://dx.doi.org/10.18488/journal.104/2015.1.1/104.1.1.11 
Faradmal, J., Soltanian, A. R., Roshanaei, G., Khodabakhshi, R., \& Kasaeian, A. (2014). Comparison of the performance of log-logistic regression and artificial neural networks for predicting breast cancer relapse. Asian Pacific Journal of Cancer Prevention: APJCP, 15(14), 5883-5888. http://dx.doi.org/10.7314/APJCP. 2014.15.14.5883

Gerds, T. A., Kattan, M. W., Schumacher, M., \& Yu, C. (2013). Estimating a time-dependent concordance index for survival prediction models with covariate dependent censoring. Statistics in Medicine, 32(13), 2173-2184. http://dx.doi.org/10.1002/sim.5681

Graf, E., Schmoor, C., Sauerbrei, W., \& Schumacher, M. (1999). Assessment and comparison of prognostic classification schemes for survival data. Statistics in Medicine, 18, 2529-2545. http://dx.doi.org/10. 1002/(SICI)1097-0258(19990915/30)18:17/18<2529::AID-SIM274>3.0.CO;2-5

Habibi, S., Ahmadi, M., \& Alizadeh, S. (2015). Type 2 Diabetes Mellitus Screening and Risk Factors Using Decision Tree: Results of Data Mining. Global Journal of Health Science, 7(5), 304-310. http://dx.doi.org/ $10.5539 /$ gjhs.v7n5p304

Haibe-Kains, B., Desmedt, C., Sotiriou, C., \& Bontempi, G. (2008). A comparative study of survival models for breast cancer prognostication based on microarray data: Does a single gene beat them all? Bioinformatics, 24(19), 2200-2208. http://dx.doi.org/10.1093/bioinformatics/btn374

Harrell, F. E., Lee, K. L., \& Mark, D. B. (1996). Multivariable prognostic models: Issues in developing models, evaluating assumptions and adequacy, and measuring and reducing errors. Statistics in Medicine, 15, 361-387. http://dx.doi.org/10.1002/(SICI)1097-0258(19960229)15:4<361::AID-SIM168>3.0.CO;2-4

Hastie, T., Tibshirani, R., Friedman, J., \& Franklin, J. (2005). The elements of statistical learning: Data mining, inference and prediction. The Mathematical Intelligencer, 27(2), 83-85. http://dx.doi.org/10.1007/BF029 85802

Heagerty, P. J., Lumley, T., \& Pepe, M. S. (2000). Time-dependent ROC curves for censored survival data and a diagnostic marker. Biometrics, 56(2), 337-344. http://dx.doi.org/10.1111/j.0006-341X.2000.00337.x

Hothorn, T., Bühlmann, P., Dudoit, S., Molinaro, A., \& Van Der Laan, M. J. (2006). Survival ensembles. Biostatistics, 7(3), 355-373. http://dx.doi.org/10.1093/biostatistics/kxj011

Hothorn, T., Hornik, K., \& Zeileis, A. (2006). Unbiased recursive partitioning: A conditional inference framework. Journal of Computational and Graphical Statistics, 15(3), 651-674. http://dx.doi.org/10.1198 /106186006X133933

Hsich, E., Gorodeski, E. Z., Blackstone, E. H., Ishwaran, H., \& Lauer, M. S. (2011). Identifying important risk factors for survival in patient with systolic heart failure using random survival forests. Circ Cardiovasc Qual Outcomes, 4(1), 39-45. http://dx.doi.org/10.1161/CIRCOUTCOMES.110.939371

Ishwaran, H. (2007). Variable importance in binary regression trees and forests. Electronic Journal of Statistics, 1(0), 519-537. http://dx.doi.org/10.1214/07-EJS039

Ishwaran, H., Kogalur, U. B., Blackstone, E. H., \& Lauer, M. S. (2008). Random survival forests. The Annals of Applied Statistics, 2(3), 841-860. http://dx.doi.org/10.1214/08-AOAS169

Kim, H., \& Loh, W.-Y. (2001). Classification trees with unbiased multiway splits. Journal of the American Statistical Association, 96(454), 598-604. http://dx.doi.org/10.1198/016214501753168271

Kurt Omurlu, I., Ture, M., \& Tokatli, F. (2009). The comparisons of random survival forests and Cox regression analysis with simulation and an application related to breast cancer. Expert Systems with Applications, 36(4), 8582-8588. http://dx.doi.org/10.1016/j.eswa.2008.10.023

Maroco, J., Silva, D., Rodrigues, A., Guerreiro, M., Santana, I., \& de Mendonça, A. (2011). Data mining methods in the prediction of Dementia: A real-data comparison of the accuracy, sensitivity and specificity of linear discriminant analysis, logistic regression, neural networks, support vector machines, classification trees and random forests. BMC Research Notes, 4(1), 1-14. http://dx.doi.org/10.1186/1756-0500-4-299

Mert, A., Kılıç, N., Bilgili, E., \& Akan, A. (2015). Breast Cancer Detection with Reduced Feature Set. Comput Math Methods Med, 11. http://dx.doi.org/10.1155/2015/265138

Michiels, S., Koscielny, S., \& Hill, C. (2005). Prediction of cancer outcome with microarrays: A multiple random validation strategy. The Lancet, 365(9458), 488-492. http://dx.doi.org/10.1016/S0140-6736(05)17866-0

Mirmohammadi, S. N., Shishehgar, M., \& Ghapanchi, F. (2014). Applications of ANNs, SVM, MDR and FR 
Methods in Bioinformatics. World Applied Sciences Journal, 31(6), 1109-1117.

Montazeri, M., Montazeri, M., Montazeri, M., \& Beigzadeh, A. (2015). Machine learning models in breast cancer survival prediction. Technology and Health Care (Preprint), 24(1), 31-42. http://dx.doi.org/10.3233/ THC-151071

Pang, H., Datta, D., \& Zhao, H. (2010). Pathway analysis using random forests with bivariate node-split for survival outcomes. Bioinformatics, 26(2), 250-258. http://dx.doi.org/10.1093/bioinformatics/btp640

Sadoughi, F., Afshar, H. L., Olfatbakhsh, A., \& Mehrdad, N. (2016). Application of Canonical Correlation Analysis for Detecting Risk Factors Leading to Recurrence of Breast Cancer. Iranian Red Crescent Medical Journal, 18(3), e23131. http://dx.doi.org/10.5812/ircmj.23131

Strobl, C., Boulesteix, A.-L., Zeileis, A., \& Hothorn, T. (2007). Bias in random forest variable importance measures: Illustrations, sources and a solution. BMC Bioinformatics, 8(1), 8-25. http://dx.doi.org/10. 1186/1471-2105-8-25

Walschaerts, M., Leconte, E., \& Besse, P. (2012). Stable variable selection for right censored data: Comparison of methods. Preprint arXiv:1203.4928.

Yosefian, I., Mosa Farkhani, E., \& Baneshi, M. R. (2015). Application of Random Forest Survival Models to Increase Generalizability of Decision Trees: A Case Study in Acute Myocardial Infarction. Comput Math Methods Med. http://dx.doi.org/10.1155/2015/576413

Zeileis, A., Hothorn, T., \& Hornik, K. (2008a). Model-based recursive partitioning. Journal of Computational and Graphical Statistics, 17(2), 492-514. http://dx.doi.org/10.1198/106186008X319331

\section{Copyrights}

Copyright for this article is retained by the author(s), with first publication rights granted to the journal.

This is an open-access article distributed under the terms and conditions of the Creative Commons Attribution license (http://creativecommons.org/licenses/by/3.0/). 